\title{
TORUS BUNDLES OVER A TORUS
}

\section{R. S. PALAIS AND T. E. STEWART}

1. If a compact Lie group $P$ acts on a completely regular topological space $E$ then $E$ is said to be a principal $P$-bundle if whenever the relation $p x=x$ holds for $p \in P, x \in E$ it follows that $p=e$, the identity of $P$. The orbit space $X=E / P$ is called the base space and the map $\pi: E \rightarrow X$ carrying $y$ into its orbit $P \cdot y$ is called the projection. Suppose now that $G$ is a Lie group, $H_{1}$ a closed subgroup of $G$ and $H$ a closed, normal subgroup of $H_{1}$ such that $P=H_{1} / H$ is compact. Then $E=G / H$ becomes a principal $P$-bundle with base space $X=G / H_{1}$ and projection $g H \rightarrow g H_{1}$ under the action $p(g H)=g p^{-1} H$. Such a principal bundle will be called canonical.

The purpose of this note is to show that if $X$ is a torus of dimension $n$ and $P$ a torus of dimension $m$ then every principal $P$-bundle over $X$ is canonical and further that the group $G$ lies in an extremely narrow class. Roughly speaking, our method is to try to lift the action of euclidean $n$-space up to the total space of the bundle and observe what obstructs this effort.

The torus of dimension $k$ will be denoted by $T^{k}$, the corresponding euclidean space by $R^{k}$. We view $R^{k}$ as acting transitively on $T^{k}$ with the lattice of integral points in $R^{k}$ acting ineffectively. We denote by $p: R^{k} \rightarrow T^{k}$ the usual covering map. Without loss of generality we assume the $T^{m}$-bundles over $T^{n}$ are differentiable. $\mathfrak{L}(X)$ will denote the Lie algebra of infinitely differentiable vector fields on $X$.

Exactly how narrow the class of Lie groups that $G$ lies in will be left to Theorem 2. For the present we prove:

Theorem 1. Suppose we have a principal bundle over $T^{n}$ with structural group $T^{m}$, total space $E, \pi: E \rightarrow T^{n}$ the projection, and $e_{0} \in \pi^{-1}(0)$. Then $E$ is acted on transitively by a 2-step nilpotent Lie group $G$. Further if $\alpha: G \rightarrow E$ is defined by $\alpha(g)=g \cdot e_{0}$ then we have a homomorphism $\beta: G \rightarrow R^{n}$, and a commutative diagram

$$
G \stackrel{\alpha}{\rightarrow} E
$$

$$
\begin{aligned}
& \beta \downarrow \quad \downarrow \pi \\
& R^{n} \underset{p}{\rightarrow} T^{n}
\end{aligned}
$$

Received by the editors March 9, 1960. 
PRoof. Let $\mathbb{S}_{k}$ denote the Lie algebra of the Lie group $R^{k}$. Since $(E, \pi)$ is a principal bundle we may consider $T^{m}$ acting on $E$ without fixed points. We have then an injective algebra homomorphism $\alpha_{1}: \mathbb{S}_{m} \rightarrow \mathfrak{R}(E)$ such that if $Z \neq 0$ then $\alpha_{1}(Z)_{p} \neq 0, p \in E$, where $\alpha_{1}(Z)_{p}$ denotes $\alpha_{1}(Z)$ evaluated at $p[2$, p. 16]. And, of course, we have the usual injective homomorphism $\phi_{1}: \mathfrak{B}_{n} \rightarrow \mathfrak{R}\left(T^{n}\right), \phi_{1}(X)_{x} \neq 0$ for $X \neq 0$, $x \in T^{n}$. We suppose now we are given a connection in $(E, \pi)[2$, p. 25]. This may be considered a linear map $\lambda: \mathbb{R}\left(T^{n}\right) \rightarrow \mathfrak{R}(E)$ such that $\left[\lambda(X), \alpha_{1}(Z)\right]=0$, and $\delta \pi(\lambda(X))=X$ where $\delta \pi$ denotes the differential of the mapping $\pi$. We identify $X \in\left(S_{n}\right.$ and $\phi_{1}(X)$ and denote $\lambda(X)$ by $X^{*}$, similarly we identify $Z \in \oiint_{m}$ with $\alpha_{1}(Z)$.

Let $\left\{X_{i}\right\}_{i=1}^{n}$ be a basis of $\mathcal{B}_{n}, X_{i}=\left(\delta_{i j}\right)_{j=1}^{n}$, and $\left\{Z_{k}\right\}$ a basis of $\oiint_{m}$. For $X, Y \in\left(\mho_{n}\right.$ we have $\delta \pi\left[X^{*}, Y^{*}\right]_{x}=\left[\delta \pi\left(X^{*}\right), \delta \pi\left(Y^{*}\right)\right]_{x}=[X, Y]_{x}$ $=0$, hence $\left[X^{*}, Y^{*}\right]_{p}=\Phi_{p}(X, Y)=\sum a^{k}(X, Y, \cdot p)\left(Z_{k}\right)_{p}$. It is easily seen that $\Phi_{p}(X, Y)$ is actually a function of $\pi(p)$ since $\left[X^{*}, Z\right]_{p}=0$, $Z \in \mathscr{G}_{m}$, i.e., $\Phi_{x}(X, Y)$ is an exterior two form on $T^{n}$. In particular we set $\Phi_{x}\left(X_{i}, \quad X_{j}\right)=\sum_{k=1}^{m} a_{i j}^{\mathbf{k}}(x)\left(Z_{k}\right)$. Now $\left.\quad\left[\begin{array}{ll}X_{i}^{*}, & X_{j}^{*}\end{array}\right], X_{r}^{*}\right]_{p}$ $=\left[\sum a_{i j}^{k}(\pi(p)) Z_{k}, X_{r}^{*}\right]_{p}=-\sum_{k=1}^{n}\left(X_{r}\left(a_{t j}^{k}(x)\right)\right) Z_{k}$. Applying the Jacobi identity $\left[\left[X_{i}^{*}, X_{j}^{*}\right], X_{r}^{*}\right]_{p}+\left[\left[X_{j}^{*}, X_{r}^{*}\right], X_{i}^{*}\right]_{p}+\left[\left[X_{r}^{*}, X_{i}^{*}\right], X_{j}^{*}\right]_{p}=0$ we obtain for $k=1, \cdots, m$

$$
X_{r}\left(a_{i j}^{k}(x)\right)+X_{i}\left(a_{j r}^{k}(x)\right)+X_{j}\left(a_{r i}^{k}(x)\right)=0 .
$$

If $\left\{\omega_{i}\right\}_{i=1}^{n}$ is the set of exterior one forms dual to $X_{i}$, i.e. $\left(\omega_{i}\left(X_{j}\right)\right)_{p}$ $=\delta_{i j}$ then equation 1.2 states that $\Phi_{x}^{k}=\left(\sum_{i<j} a_{i j}^{k} \omega_{i} \wedge \omega_{j}\right)_{x}$ is a closed form, i.e., $d \Phi^{k}=0$, Now as is well known every closed form of the torus is cohomologous to an invariant form $[1$, p. 95]. Hence for each $k$ there exists a two form $\Psi^{k}$ on $T^{n}$ which does not vary with $x \in T^{n}$, and a one form $\theta^{k}$ on $T^{n}$ such that

$$
\Phi^{k}-\Psi^{k}=d \theta^{k} .
$$

In terms of our chosen basis we have a set of constants $c_{i j}^{k}$ and a set of functions $b_{r}^{k}(x)$ such that for $i, j, k$

$$
\stackrel{k}{a_{i j}}(x)-\stackrel{k}{c_{i j}}=X_{j}\left(b_{i}^{k}(x)\right)-X_{i}\left(b_{j}^{k}(x)\right) .
$$

For each $i$, set $X_{i}^{* *}=X_{i}^{*}+\sum_{k=1}^{m} b_{i}^{k}(x) Z_{k}$. It follows easily from (1.4) that

$$
\left[X_{i}^{* *}, X_{j}^{* *}\right]_{p}=\sum_{k=1}^{m} c_{i j}^{k}\left(Z_{k}\right)_{p} .
$$

Extending the function $X_{i} \rightarrow X_{i}^{* *}$ linearly over $B_{n}$ we obtain a linear map $\mathfrak{S}_{n} \rightarrow \mathbb{R}(E)$ satisfying 


$$
\begin{aligned}
{\left[X^{* *}, Y^{* *}\right]_{p} } & =\sum_{k=1}^{m} \Psi^{k}(X, Y)\left(Z_{k}\right)_{p}, \\
\delta \pi\left(X^{* *}\right) & =X .
\end{aligned}
$$

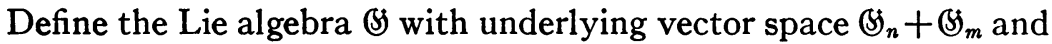
bracket operation defined by

$$
\begin{aligned}
& {[X, Z]=0, \quad X \in \mathfrak{S}_{,}, Z \in \mathbb{S}_{m},} \\
& {[X, Y]=\sum_{k} \Psi^{k}(X, Y) Z_{k}, \quad X, Y \in \mathbb{S}_{n} .}
\end{aligned}
$$

According to (1.6) and (1.7) we have an isomorphism $\chi: B \rightarrow ?(E)$ such that $(\chi(X))_{p} \neq 0$ for $X \neq 0, p \in E$. If $G$ is the simply connected Lie group associated to $B$ it follows from the compactness of $E$ that we can integrate the isomorphism $\chi$ to obtain an action of $G$ on $E$. Since each orbit will be open and $E$ is connected this action is transitive. $G$ is nilpotent since $B$ is and $\beta: G \rightarrow R^{n}$ is the homomorphism associated to the Lie algebra homomorphism $X+Z \rightarrow X, X \in \mathcal{S}_{n}$, $Z \in \mathcal{S}_{m}$. The diagram (1.1) follows easily, and hence the theorem.

For notational purposes we restrict ourselves now to the case $m=1$. The following statements are easily generalized to the case $m>1$.

Let $Z$ be a unit vector of $\xi_{1}$. The Lie algebra $(S)$ defined in the last theorem is given by the space $\mathfrak{B S}_{n}+\mathfrak{S}_{1}$ with

$$
\begin{aligned}
& {[X, Z]=0, \quad X \in \oiint, Z \in \mathfrak{B}_{1},} \\
& {[X, Y]=\Psi(X, Y) Z, \quad X, Y \in \mathfrak{B}_{n},}
\end{aligned}
$$

$\Psi$ bilinear and skew-symmetric.

We describe now the group $G$. As a space we have clearly $G=R^{n}$ $\times R^{1}$. As one can readily verify (or else by the Baker-Hausdorff formula) the multiplication in $G$ is given by

$$
(x, y) \cdot\left(x^{\prime}, y^{\prime}\right)=\left(x+x^{\prime}, y+y^{\prime}+\frac{1}{2} \Psi\left(x, x^{\prime}\right)\right) .
$$

We have here identified $\mathfrak{S}_{k}$ and $R^{k}$. We have then:

THEOREM 2. If both $x$ and $x^{\prime}$ belong to the lattice of integers in $R^{n}$, then $\Psi\left(x, x^{\prime}\right)$ is integral.

Proof. We show first that if $x$ is in the lattice of integers in $R^{n}$ then there exists $y \in R^{1}$ such that $(x, y)$ is in the isotropy group of $e_{0} \in \pi^{-1}(0)$.

Indeed it follows from the commutativity of the diagram (1.1) that $(x, 0) \cdot e_{0} \in \pi^{-1}(0)$ and hence there is $y \in R^{1}$ such that $e_{0}=(0, y)\left((x, 0) \cdot e_{0}\right)=(x, y) \cdot e_{0}$. 
Now let $x$ and $x^{\prime}$ be in the lattice of integers in $R^{n}$ and $y, y^{\prime}$ elements of $R^{1}$ such that $(x, y)$ and $\left(x^{\prime}, y^{\prime}\right)$ are in the isotropy group $G_{e_{0}}$ of $e_{0}$. Then the commutator of these two elements is in $G_{e_{0}}$. Since $(x ; y)^{-1}=(-x,-y)$ we have

$$
(x, y)\left(x^{\prime}, y^{\prime}\right) \cdot(x, y)^{-1} \cdot\left(x^{\prime}, y^{\prime}\right)^{-1}=\left(0, c\left(x, x^{\prime}\right)\right) \in G_{e_{0}} .
$$

Since $(0, y) \in G_{\theta_{0}}$ if and only if $y$ is integral the theorem follows.

It should be noted that Theorem 1 is not true if the torus is only a fibre and not actually the structural group. For example, the two dimensional Klein bottle is not acted on transitively by a nilpotent group, but is the total space of a bundle over the circle with circle as fibre.

2. The authors would like to express their appreciation to the referee for pointing out the following theorem which strengthens Theorem 2.

THEOREM 3. A space $X$ is a compact 2-step nilmanifold if and only if it is the total space of a principal $T^{m}$-bundle over $T^{n}$.

Proof (Following the Referee). The sufficiency follows from Theorem 1 . Now suppose $X$ is acted on transitively by a connected, simply connected 2-step nilpotent group, $N$, with stability group $\Gamma$ which is discrete $[2, \mathrm{p} .12]$. Set $P=[N, N]$, the commutator group of $N$, and $G=P \cdot \Gamma$. Then $P$ is central since $N$ is a 2-step nilpotent group and $\Gamma$ is invariant in $G$, and $G$ is invariant in $N$. Since $N / G$ and $G / \Gamma$ are then compact, connected, abelian Lie groups, they are tori. Then the assertion follows from the bundle $X=N / \Gamma \rightarrow N / G$.

\section{REFERENCES}

1. C. Chevalley and S. Eilenberg, Cohomology theory of Lie groups and Lie algebras, Trans. Amer. Math. Soc. vol. 63 (1948) pp. 85-124.

2. A. I. Malcev, On a class of homogeneous spaces, Amer. Math. Soc. Translations ser. 1 , no. $39,1951$.

3. K. Nomizu, Lie groups and differential geometry, Mathematical Society of Japan, 1956.

INSTITUTE FOR AdVANCEd StUdy 